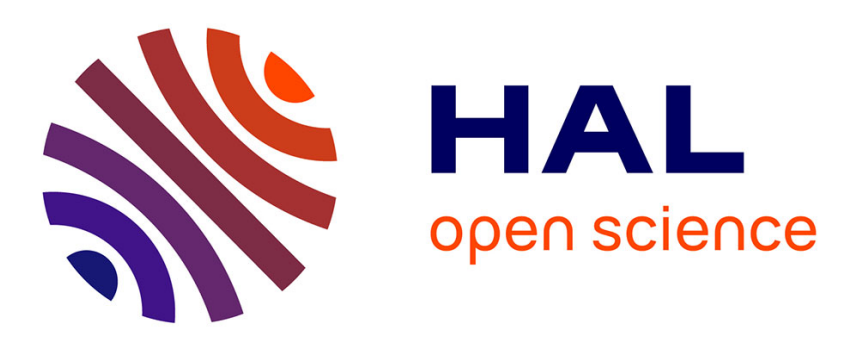

\title{
Robust Output Interval Constraint using O/I Saturation Transformation with Application to Uncertain Linear Launch Vehicle
}

\author{
Emmanuel Chambon, Laurent Burlion, Pierre Apkarian
}

\section{- To cite this version:}

Emmanuel Chambon, Laurent Burlion, Pierre Apkarian. Robust Output Interval Constraint using O/I Saturation Transformation with Application to Uncertain Linear Launch Vehicle. Proceedings of the 14th European Control Conference, Jul 2015, Linz, Austria. pp.7. hal-01208420

\section{HAL Id: hal-01208420 \\ https://hal.science/hal-01208420}

Submitted on 2 Oct 2015

HAL is a multi-disciplinary open access archive for the deposit and dissemination of scientific research documents, whether they are published or not. The documents may come from teaching and research institutions in France or abroad, or from public or private research centers.
L'archive ouverte pluridisciplinaire HAL, est destinée au dépôt et à la diffusion de documents scientifiques de niveau recherche, publiés ou non, émanant des établissements d'enseignement et de recherche français ou étrangers, des laboratoires publics ou privés. 


\title{
Robust Output Interval Constraint using O/I Saturation Transformation with Application to Uncertain Linear Launch Vehicle
}

\author{
Emmanuel Chambon ${ }^{\star}$, Laurent Burlion ${ }^{\star}$ and Pierre Apkarian ${ }^{\star}$
}

\begin{abstract}
In this article, we consider the case where a regulated output of an uncertain system with unknown disturbance input is constrained to lie between two specified timevarying bounds. We suppose a controller has already been designed using any desired technique. This controller may not satisfy expected output constraints. The objective is to design saturations on the control signal so that the closed-loop is altered only when those specifications are not met. The theory is presented in the linear case with linear dependence on an unknown disturbance. Application to an uncertain launcher linear model with unknown wind disturbance is presented.

Index Terms-Linear systems; Constrained control; Uncertain systems; Unknown input.
\end{abstract}

\section{INTRODUCTION}

The objective of control law synthesis is often to constrain time-response of a so-called regulated variable. Characteristics like overshoot or settling time should satisfy prescribed behavior. Regulated variables however may not be measured or may depend on unknown disturbance. Additional information is thus required.

In this article we consider the case where we want to force a system regulated output variable $\alpha$ to lie in the possibly time-varying interval $\left[\alpha_{\min }(t), \alpha_{\max }(t)\right]$. This variable linearly depends on the system state but also on an unknown input $d_{w}$ through

$$
\alpha=C_{\alpha} x+D_{\alpha}\left(d_{w} u\right)^{\top}
$$

where $\left(C_{\alpha}, D_{\alpha}\right)$ are known. Uncertainties in the plant model are also considered. We suppose we can bound unknown input by deterministic time-varying signals. Uncertainty ranges also are supposed known. A controller has been synthesized which robustly stabilizes the system. The interval constraint on the regulated output variable $\alpha$ is however not satisfied.

Some strategies to answer this problem include [1], [2] which use anti-windup loops to constrain the state or outputs in the time-domain. In this article however, the recent work in [3] is used to transform such constraint on a regulated output into time-varying saturations on the control signal. In the presence of uncertainties and unknown inputs, calculating these saturations however requires additional study. We propose a robust approach where the regulated variable is estimated through the use of a Luenberger observer. Estimation error - which depends on disregarded uncertainties and disturbance inputs - is then bounded using interval observers as described in [4], [5]. Although being conservative, this

\footnotetext{
${ }^{\star}$ Onera - The French Aerospace Lab, F-31055 Toulouse, France, Emmanuel.Chambon@onera.fr
}

approach has been successfully tested on an uncertain rigid launch vehicle model with unknown wind input. Other successful applications of Output to Input Constraint Transformation (OIST) include visual servoing applied to a loworder known UAV model [6] and longitudinal load control of an aircraft [7] where a known linear large-scale model is considered.

The paper is structured as follows. Section II introduces notations and formulates the problem as well as the considered hypotheses. Section III provides theoretical results related to OIST. In Section IV we describe our solution which involves the use of different types of observers. Our approach is then applied to a rigid launch vehicle model in Section V. Conclusions and perspectives are given in Section VI.

\section{THE OIST PROBLEM}

\section{A. Notations and a definition}

Let $\mathbb{R}$ (resp. $\mathbb{N}$ ) denote the set of real numbers (resp. of natural integers). Let $A \in \mathbb{R}^{n \times m}$. The Moore-Penrose pseudoinverse of $A$ is denoted $A^{\dagger}$. For a matrix $A(\Theta)$ depending on an uncertain vector $\Theta \in \mathbb{R}^{r}$ with known nominal value $\Theta_{\text {nom }}$, notation $A$ refers to $A\left(\Theta_{\text {nom }}\right)$. Let $r \in \mathbb{N}, \bar{r}$ refers to $\{1,2, \ldots, r\}$. The identity matrix of size $n \times n$ is written $I_{n}$.

Inequalities over matrices are to be understood elementwise: considering $A=\left(a_{i j}\right)$ and $B=\left(b_{i j}\right)$ in $\mathbb{R}^{n \times m}, A \leq$ $B \Leftrightarrow \forall i \in \bar{n}, j \in \bar{m}, a_{i j} \leq b_{i j}$. For a matrix $A \in \mathbb{R}^{n \times m}$ we denote $A^{u}=\max (A, 0)$ (where max is the component-wise maximum function) and $A^{l}=A^{u}-A$. Kronecker delta is denoted $\kappa(i, j)=\kappa_{i, j}$ and is null unless $i=j$.

Definition 1: Let $A \in \mathbb{R}^{n \times n}$. $A$ is said to be Metzler iff

$$
\forall i \neq j \in \bar{n}, a_{i j} \geq 0
$$

\section{B. Hypotheses}

The hypotheses satisfied by the systems considered throughout the article are regrouped here.

Hypothesis 1: the disturbance signal $d_{w} \in \mathbb{R}$ is unknown but time-varying bounds $M(t)$ (resp. $M_{2}(t)$ ) are known such that $\forall t,\left|d_{w}(t)\right| \leq M(t)\left(\operatorname{resp} .\left|\dot{d}_{w}(t)\right| \leq M_{2}(t)\right)$.

Hypothesis 2: $y=x$ and $\alpha=C_{\alpha} x$ is of relative degree $k \in \mathbb{N}^{+}$with respect to $u$, and relative degree $0<l \leq k$ with respect to $d_{w}$ that is

$$
\left\{\begin{array}{llll}
\forall i<k-1, & C_{\alpha} A^{i} B_{u}=0 & \& & C_{\alpha} A^{k-1} B_{u} \neq 0 \\
\forall i<l-1, & C_{\alpha} A^{i} B_{d}=0 & \& & C_{\alpha} A^{l-1} B_{d} \neq 0
\end{array}\right.
$$

It is also supposed that $C_{\alpha} A^{k-1} B_{u}>0$. 
Hypothesis 3: the relative degrees wrt. $u$ and $d$ satisfy to the following relation: $k=l+1$.

Hypothesis 4: As far as initial values are considered, we have $\alpha_{\min } \leq \alpha(0) \leq \alpha_{\max }$ and

$$
\forall j \in \overline{k-1}, \alpha_{j, \min }(0) \leq \alpha^{(j)}(0) \leq \alpha_{j, \max }(0)
$$

where $\alpha_{j, \min }$ and $\alpha_{j, \max }$ are defined recursively in Fig. 1 with $\alpha_{0, \min }=\alpha_{\min }$.

Hypothesis 5: Suppose there exists a matrix $R$ such that $M=R(A-L C) R^{-1}=R A_{e} R^{-1}$ is Hurwitz Metzler.

Hypothesis 6: Suppose $\left|C_{\alpha} A^{k-1} B_{d} d_{w}\right| \gg K_{k}\left|C_{\alpha} A^{k-2} B_{d} d_{w}\right|$ (where $K_{k}>0$ is fixed) and $\left|C_{\alpha} A^{k-1} B_{d} d_{w}\right| \gg\left|C_{\alpha} A^{k-2} B_{d} \dot{d}_{w}\right|$

\section{Problem formulation}

Let $\Theta=\left\{\theta_{i}\right\}_{i \in \bar{r}}$ a vector of uncertain parameters satisfying $\forall i \in \bar{r}, \exists\left(\underline{\theta_{i}}, \overline{\theta_{i}}\right) \in \mathbb{R}^{2}$ s.t. $\underline{\theta_{i}} \leq \theta_{i} \leq \overline{\theta_{i}}$. By extent, we define $\underline{\Theta}=\left(\underline{\theta_{i}}\right)_{i} \in \mathbb{R}^{r}$ and $\bar{\Theta}$ similarly. The notation $\Theta_{\text {nom }}$ is used to refer to known nominal value of these parameters. We consider the following uncertain linear system

$$
(G)\left\{\begin{aligned}
\dot{x} & =A(\Theta) x+B_{u}(\Theta) u+B_{d}(\Theta) d_{w} \\
y & =C x
\end{aligned}\right.
$$

where $x \in \mathbb{R}^{n}, y \in \mathbb{R}^{m}, u \in \mathbb{R}$, matrices $\left(A, B_{u}, B_{d}\right)$ dependency on the vector of uncertain parameters $\Theta$ is known, $C$ is known and $d_{w} \in \mathbb{R}$ is an unknown disturbance with time-varying lower and upper bounds

$$
\forall t, \underline{d}_{w}(t) \leq d_{w}(t) \leq \bar{d}_{w}(t)
$$

We suppose a stabilizing static or dynamic control law $u$ has been designed using any linear control design technique like $H_{\infty}$ synthesis or modal control. We consider the following problem

Problem 1: Find $u_{\min }, u_{\max }$ and $\mathbf{C}_{0}$ such that

$$
\alpha(t) \in\left[\alpha_{\min }(t), \alpha_{\max }(t)\right], \forall t
$$

for the system described as

$$
\left\{\begin{aligned}
\dot{x} & =A(\Theta) x+B_{u}(\Theta) u+B_{d}(\Theta) d_{w} \\
y & =C x \\
\alpha & =C_{\alpha} x+D_{\alpha}\left(\begin{array}{c}
d_{w} \\
u
\end{array}\right) \in \mathbb{R} \\
u & \in\left[u_{\min }\left(\underline{d}_{w}, \bar{d}_{w}, \underline{\Theta}, \bar{\Theta}, \alpha_{\min }, \alpha_{\max }, y\right),\right. \\
& \left.u_{\max }\left(\underline{d}_{w}, \bar{d}_{w}, \underline{\Theta}, \bar{\Theta}, \alpha_{\min }, \alpha_{\max }, y\right)\right] \\
x_{0} & \in \mathbf{C}_{0}
\end{aligned}\right.
$$

Apart from being expressed in the linear framework, this is a slightly different problem from the Robust Output Constraint Problem proposed in [3] since the regulated variable $\alpha$ linearly depends on the unknown disturbance $d_{w}$ and the considered system depends on parameters $\Theta$. Note however that the specified bounds $\alpha_{\min }(t)$ and $\alpha_{\max }(t)$ do not depend on this vector $\Theta$.

\section{Uncertainties viewed as nominal system inputs}

In robust control theory, uncertainties are commonly injected into a system through Linear Fractional Transformation (LFT) with respect to a block $\Delta \in \mathbb{R}^{r \times r}$ (see [8]). This means that system (5) is equivalent to a known (nominal) system perturbed by inputs $d_{\Delta}$ due to the presence of uncertainties. These inputs themselves depend on the other system variables through

$$
\left(G_{u}\right) \begin{cases}\dot{x} & =A x+B\left(\begin{array}{ll}
d_{w} & u
\end{array}\right)^{\top}+B_{\Delta} d_{\Delta} \\
d_{\Delta} & =\Delta z \\
z & =C_{\Delta} x+D_{\Delta 1}\left(\begin{array}{ll}
d_{w} & u
\end{array}\right)^{\top}+D_{\Delta 2} d_{\Delta} \\
y & =C x\end{cases}
$$

where $B=\left[B_{d}\left(\Theta_{\text {nom }}\right) \quad B_{u}\left(\Theta_{\text {nom }}\right)\right], z \in \mathbb{R}^{r}$ and $\Delta$ is a diagonal matrix with elements $\delta_{i}(i \in \bar{r})$ satisfying $\left|\delta_{i}\right| \leq 1$. When comparing Eq. (5) and Eq. (9), one can see that $B_{\Delta} d_{\Delta}$ contains information on the variation of uncertain parameters $\Theta$ around their nominal value $\Theta_{\text {nom. This formulation will }}$ be used in the decoupling of $\alpha$ with respect to $d_{w}$ and $u$ (see Section II-E). Also, problem 2 will be reformulated in presence of uncertainties by addition of a disturbance input $d_{\Delta}$ to the existing input $d_{w}$.

\section{E. Unknown input decoupling}

To use the methods proposed in [3], we can artificially decouple $\alpha$ and $d_{w}$. Assuming $B$ is full-column rank, we have $B^{\dagger} B=I_{2}$. Using Eq. (9), we have $\dot{x}=A x+B\left(\begin{array}{ll}d_{w} & u\end{array}\right)^{\top}+B_{\Delta} d_{\Delta}$ hence

$$
\left(\begin{array}{ll}
d_{w} & u
\end{array}\right)^{\top}=B^{\dagger} \dot{x}-B^{\dagger} A x-B^{\dagger} B_{\Delta} d_{\Delta}
$$

Since $\alpha=C_{\alpha} x+D_{\alpha}\left(\begin{array}{ll}d_{w} & u\end{array}\right)^{\top}$, we have

$$
\begin{aligned}
\alpha & =C_{\alpha} x+D_{\alpha} B^{\dagger}\left(\dot{x}-A x-B_{\Delta} d_{\Delta}\right) \\
& =\Sigma_{1} x+\Sigma_{2} \dot{x}-D_{\alpha} B^{\dagger} B_{\Delta} d_{\Delta} \\
& =\Sigma(s) x-D_{\alpha} B^{\dagger} B_{\Delta} d_{\Delta}
\end{aligned}
$$

with the notations

$$
\begin{array}{ll}
\Sigma_{1} & :=C_{\alpha}-D_{\alpha} B^{\dagger} A \\
\Sigma_{2} & :=D_{\alpha} B^{\dagger} \\
\Sigma(s) & :=\Sigma_{1}+\Sigma_{2} s
\end{array}
$$

Note this requires a knowledge of the state $x$ through observation in case $y \neq x$. Also note that $\Sigma(s)$ is not proper but $T_{\alpha \rightarrow \xi}(s) \Sigma(s)$ may be where $\xi:=T_{\alpha \rightarrow \xi}(s) \alpha$ is an appropriate change of variable. Note a similar change of variable is considered later in this article for reasons detailed in IV-D.1. If $T_{\alpha \rightarrow \xi}(s) \Sigma(s)$ is proper, an estimation of $\dot{x}$ is not required. Otherwise, specific techniques need to be used like high-gain observers [9] or algebraic time-derivative estimation [10] which is not considered in this article.

\section{O/I SATURATION TRANSFORMATION}

For simplicity of the presentation, we only describe application of OIST to the known linear perturbed case. Please refer to [3] for a comprehensive discussion. In case the model is uncertain, consider an additional input $d_{\Delta}$ as in problem 1 using Eq. (9). Consider the following problem

Problem 2: Find $u_{\min }, u_{\max }$ and $\mathbf{C}_{0}$ such that 


$$
\alpha(t) \in\left[\alpha_{\min }(t), \alpha_{\max }(t)\right], \forall t
$$

for the system described as

$$
\left\{\begin{array}{l}
\dot{x}=A x+B_{u} u+B_{d} d_{w} \\
y=C x \\
\alpha=C_{\alpha} x \\
u \quad \in\left[u_{\min }\left(y, \alpha_{\min }, \alpha_{\max }\right), u_{\max }\left(y, \alpha_{\min }, \alpha_{\max }\right)\right] \\
x_{0} \in \mathbf{C}_{0}
\end{array}\right.
$$

where $x \in \mathbb{R}^{n}, y \in \mathbb{R}^{m}, u \in \mathbb{R}$ and system matrices are known. Under Hyp. 1 to 4 we formulate the theorem

Theorem 1: Let $\left(K_{1}, \ldots, K_{k}\right)$ fixed positive real numbers. Let Hyp. 2 to 4 be satisfied. We have

$$
\alpha^{(k)}=C_{\alpha} A^{k} x+C_{\alpha} A^{k-1} B_{u} u+C_{\alpha} A^{k-1} B_{d} d_{w}+C_{\alpha} A^{k-2} B_{d} \dot{d}_{w}
$$

Requirements $\alpha(t) \in\left[\alpha_{\min }(t), \alpha_{\max }(t)\right], \forall t$ and $\forall m \in$ $\bar{k}, \alpha_{m, \min } \leq \alpha^{(m)} \leq \alpha_{m, \max }$ (where bounds are defined in Eq. (16) and (17) in Fig. 1) are satisfied under application of the following saturations on control signal $u$

$$
u_{\min }=\frac{\alpha_{k, \min }\left(x, \dot{x}, d_{w}\right)-C_{\alpha} A^{k} x-C_{\alpha} A^{k-1} B_{d} d_{w}-C_{\alpha} A^{k-2} B_{d} \dot{d}_{w}}{C_{\alpha} A^{k-1} B_{u}}
$$

with $u_{\max }$ defined in a similar way. Under Hyp. 1 a more restrictive expression is obtained

$$
\begin{array}{r}
u_{\min }=\frac{1}{C_{\alpha} A^{k-1} B_{u}}\left(\alpha_{k, \min }(x, \dot{x}, 0)-C_{\alpha} A^{k} x+\left[\left|C_{\alpha} A^{k-1} B_{d}\right|\right.\right. \\
\left.\left.+K_{k}\left|C_{\alpha} A^{k-2} B_{d}\right|\right] M+\left|C_{\alpha} A^{k-2} B_{d}\right| M_{2}\right)
\end{array}
$$

Note that $\left(K_{i}\right)_{i} \in \bar{k}$ static coefficients in $\alpha_{k, \min }(x, \dot{x}, 0)$ need to be chosen wisely to avoid saturations crossings.

Proof: due to space restriction, the proof of this theorem is not detailed here. A sketch of the proof in presence of disturbance is proposed in [3, p. 1219].

\section{PROPOSED SOLUTION}

The regulated variable $\alpha$ has been decoupled from the unknown input $d_{w}$ in Section II-E. Further knowledge is however needed since $\alpha$ depends on $(x, \dot{x})$ according to Eq. (11) which leads to $u_{\min }$ and $u_{\max }$ depending on the state and its derivative, as shown in Th. 1. In this article, we propose using an LTI Luenberger observer in parallel with an interval observer. Then we proceed to OIST application.

\section{A. State observer and error system}

We use the following observer to estimate $\widehat{x}$ based on system $G$ - see Eq. $(5)$ - nominal model $\left(\Theta=\Theta_{\text {nom }}\right)$

$$
\left(G_{\mathrm{obs}}\right)\left\{\begin{array}{l}
\dot{\hat{x}}=A \widehat{x}+B_{u} u+L(y-C \widehat{x}) \\
y_{\mathrm{obs}}=\widehat{x}
\end{array}\right.
$$

This observer is based on the nominal model and does not take unknown input $d_{w}$ into account. As such the resulting observation error depends on both $d_{w}$ and $\Theta$. Let $e=x-\widehat{x}$ and

$$
\begin{array}{ll}
\Delta A(\Theta) & :=A(\Theta)-A \\
\Delta B_{u}(\Theta) & :=B_{u}(\Theta)-B_{u}
\end{array}
$$

Using the following notations

$$
\begin{aligned}
& A_{e}(\Theta):=(A-L C)+\Delta A(\Theta) \\
& B_{e}(\Theta):=\left[\begin{array}{ll}
\Delta A(\Theta) & \Delta B_{u}(\Theta)
\end{array}\right]
\end{aligned}
$$

the estimation error dynamics is given by

$\left(G_{e}\right)\left\{\begin{aligned} \dot{e} & =A_{e}(\Theta) e+B_{e}(\Theta)(\widehat{x} u)^{\top}+B_{d}(\Theta) d_{w} \\ y_{e} & =e\end{aligned}\right.$

on which methods presented in [5] can be applied.

\section{B. Estimation error interval observer}

The purpose of such an observer is to determine $\underline{e}$ and $\bar{e}$ such that $\forall t, \underline{e}(t) \leq e(t) \leq \bar{e}(t)$. Suppose we can define $\underline{\Delta A} \leq \Delta A(\Theta) \leq \overline{\Delta A}$ with $\underline{\Delta A}=-\overline{\Delta A}^{1}$ and $\left(\Delta B_{u}^{\min }, \Delta B_{u}^{\max }\right)$ are known with $\Delta B_{u}^{\min }=-\Delta \overline{B_{u}^{\max 2}}$. Let

$$
\begin{aligned}
& \underline{B_{e}}(\widehat{x}, u):=\left[\begin{array}{ll}
-\operatorname{sgn}(\widehat{x}) \overline{\Delta A} & \operatorname{sgn}(u) \Delta B_{u}^{\min }
\end{array}\right] \\
& \overline{B_{e}}(\widehat{x}, u):=-\underline{B_{e}}(\widehat{x}, u)
\end{aligned}
$$

Eq. (23) can be written in the following form

$\left(G_{e}\right)\left\{\begin{array}{l}\dot{e}=A_{e} e+B_{e}(\Theta)(\widehat{x} u)^{\top}+B_{d}(\Theta) d_{w}+f(e, \Theta) \\ y_{e}=e\end{array}\right.$

where $A_{e}=A_{e}(0)$ and $f(e, \Theta)=\left(A_{e}(\Theta)-A_{e}\right) e=\Delta A(\Theta) e$. Since $A_{e}(\Theta)=A_{e}(0)+\Delta A(\Theta)$ we obtain

$$
A_{e}-\overline{\Delta A} \leq A_{e}(\Theta) \leq A_{e}+\overline{\Delta A}
$$

and using [5], we consider

$$
\left\{\begin{array}{l}
\underline{f}(\underline{e}, \bar{e}):=-\overline{\Delta A}(\bar{e}-\underline{e}) \\
\bar{f}(\underline{e}, \bar{e}):=-\underline{f}(\underline{e}, \bar{e})
\end{array}\right.
$$

Suppose we have $B_{d}^{\min } \leq B_{d}(\Theta) \leq B_{d}^{\max }$. We define

$$
\underline{B_{d}}(d):=\left\{\begin{array}{ll}
B_{d}^{\min } & \text { if } d \geq 0 \\
B_{d}^{\max } & \text { if } d<0
\end{array}, \overline{B_{d}}(d):= \begin{cases}B_{d}^{\max } & \text { if } d \geq 0 \\
B_{d}^{\min } & \text { if } d<0\end{cases}\right.
$$

In case $A_{e}$ is Hurwitz Metzler ${ }^{3}$, we can use the interval observer in Eq. (29) in Fig. 2 which is described in [5], [11], [4]. Since $A_{e}$ is Hurwitz but not Metzler, suppose Hyp. 5 is satisfied. Let $e_{z}=R e$. Eq. (25) reformulates as

$$
\left\{\begin{aligned}
\dot{e}_{z} & =M e_{z}+R B_{e}(\Theta)(\widehat{x} \quad u)^{\top}+R B_{d}(\Theta) d_{w} \\
& +R f\left(R^{-1} e_{z}, \Theta\right)
\end{aligned}\right.
$$

Using notations in Section II-A and Appendix A, we obtain the interval observer as defined in Eq. (30) in Fig. 2. Estimation error $e$ then satisfies $\underline{e} \leq e \leq \bar{e}$ if

\footnotetext{
${ }^{1}$ This is specific to our application (see Section V).

${ }^{2}$ This is also specific to our application. In other cases, refer to [5] and expect more difficult expressions.

${ }^{3}$ See Def. 1.
} 


$$
\begin{gathered}
\forall j \in \overline{l-1}, \alpha_{j+1, \min }(x):=K_{j+1}\left(\alpha_{j, \min }(x)-C_{\alpha} A^{j} x\right)+\dot{\alpha}_{j, \min } \\
\text { With } k=l+1, \alpha_{k, \min }\left(x, \dot{x}, d_{w}\right):=K_{k}\left(\alpha_{l, \min }-C_{\alpha} A^{l} x\right)+\dot{\alpha}_{l, \min }-K_{k} C_{\alpha} A^{k-2} B_{d} d_{w}
\end{gathered}
$$

Fig. 1. OIST (under Hyp. 3): satisfied $\alpha^{(m)}$ bounds for $m \in \bar{k}$ in which $\alpha_{l+j, \max }$ is defined similarly to $\alpha_{l+j, \min }$.

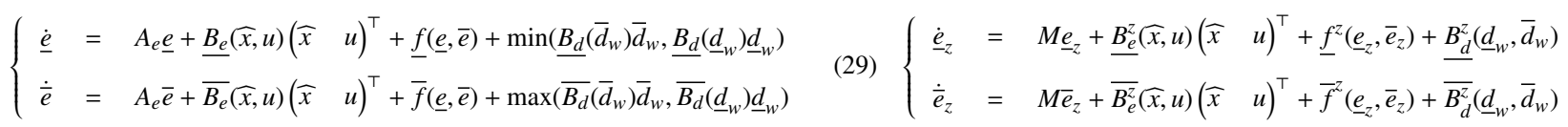

Fig. 2. Left (resp. right): valid interval observer in case $A_{e}$ (resp. $\left.M=R A_{e} R^{-1}\right)$ is Hurwitz Metzler.

$$
\left\{\begin{array}{l}
\underline{e}=T^{u} \underline{e}_{z}-T^{l} \bar{e}_{z} \\
\bar{e}=T^{u} \bar{e}_{z}-T^{l} \underline{e}_{z}
\end{array}\right.
$$

and state $x$ satisfies $\widehat{x}+\underline{e} \leq x \leq \widehat{x}+\bar{e}$.

\section{Regulated variable estimation error}

Recall that $\alpha=\Sigma_{1} x+\Sigma_{2} \dot{x}-D_{\alpha} B^{\dagger} B_{\Delta} d_{\Delta}$. Let $e_{\alpha}=\alpha-\widehat{\alpha}$ where $\widehat{\alpha}=\Sigma_{1} \widehat{x}+\Sigma_{2} \dot{\hat{x}}$. Thus

$$
\begin{aligned}
e_{\alpha} & =\Sigma_{1} e+\Sigma_{2} \dot{e}-D_{\alpha} B^{\dagger} B_{\Delta} d_{\Delta} \\
& =e_{\alpha}^{1}+e_{\alpha}^{2} \\
\underline{e}_{\alpha} & =\underline{e}_{\alpha}^{1}+\underline{e}_{\alpha}^{2} \\
\bar{e}_{\alpha} & =\bar{e}_{\alpha}^{1}+\bar{e}_{\alpha}^{2} \\
e_{\alpha}^{1} & =\Sigma_{1} e+\Sigma_{2} \dot{e} \\
e_{\alpha}^{2} & =-D_{\alpha} B^{\dagger} B_{\Delta} d_{\Delta}
\end{aligned}
$$

which we can bound using previous results

$$
\begin{aligned}
& \underline{e}_{\alpha}^{1}=\Sigma_{1}^{u} \underline{e}-\Sigma_{1}^{l} \bar{e}+\min \left(\Sigma_{2}^{u} \dot{\bar{e}}-\Sigma_{2}^{l} \dot{e}, \Sigma_{2}^{u} \dot{\dot{e}}-\Sigma_{2}^{l} \dot{\bar{e}}\right) \\
& \bar{e}_{\alpha}^{1}=\Sigma_{1}^{u} \bar{e}-\Sigma_{1}^{l} \underline{e}+\max \left(\Sigma_{2}^{u} \dot{\bar{e}}-\Sigma_{2}^{l} \underline{\dot{e}}, \Sigma_{2}^{u} \underline{\dot{e}}-\Sigma_{2}^{l} \dot{\bar{e}}\right) \\
& \left.\underline{e}_{\alpha}^{2}=\min \left[\begin{array}{lll}
\mathcal{B}\left(\underline{d}_{\delta_{1}}\right. & \ldots & \underline{d}_{\delta_{r}}
\end{array}\right)^{\top}, \ldots, \mathcal{B}\left(\bar{d}_{\delta_{1}} \quad \ldots \quad \bar{d}_{\delta_{r}}\right)^{\top}\right] \\
& \left.\bar{e}_{\alpha}^{2}=\max \left[\begin{array}{lll}
\mathcal{B}\left(\underline{d}_{\delta_{1}}\right. & \ldots & \underline{d}_{\delta_{r}}
\end{array}\right)^{\top}, \ldots, \mathcal{B}\left(\bar{d}_{\delta_{1}} \quad \ldots \quad \bar{d}_{\delta_{r}}\right)^{\top}\right]
\end{aligned}
$$

where $\mathcal{B}=-D_{\alpha} B^{\dagger} B_{\Delta}$ and $d_{\delta_{i}}(i \in \bar{r})$ extrema are functions of $\delta_{i}$ and $\theta_{i \text {,nom }}$ (see for example V-B). These bounds take into account the fact we use the estimated state (and estimated state derivative) to compute an estimate of regulated variable $\widehat{\alpha}$ (cf. $e_{\alpha}^{1}$ ) and that the model we use is nominal (cf. $e_{\alpha}^{2}$ ).

\section{Robust OIST}

Let $\alpha_{n}=\Sigma_{1} x+\Sigma_{2} \dot{x}$. Note that $\alpha=\alpha_{n}+e_{\alpha}^{2}$. Suppose $\alpha_{n}=T_{u \rightarrow \alpha_{n}}(s) u$ is of relative degree $k \in \mathbb{N}^{+}$with respect to $u$ and $l=k-1$ with respect to $d_{w}$.

1) Change of variable (optional): In case $T_{u \rightarrow \alpha_{n}}(s)$ has $n_{\text {unst }}$ unstable poles with $n_{\text {unst }}>k$, we define a stable and strictly proper change of variable $\xi=T_{\alpha_{n} \rightarrow \xi} \alpha_{n}$ of degree $n_{\text {unst }}-k$ and we proceed with the study of $\xi=T_{\alpha_{n} \rightarrow \xi} T_{u \rightarrow \alpha_{n}} u=$ $T_{u \rightarrow \xi} u$. Note $\xi$ is of relative degree $k_{\xi}=n_{\text {unst }}$ with respect to $u$. In case a change of variable is not needed, this is equivalent to taking $\xi=\alpha_{n}$.
2) Transfer study: Consider the transfer function $T_{u \rightarrow \xi}(s)$. We define $T_{\xi}(s)$ and $T_{u}^{\star}(s)$ such that:

$$
T_{\xi}(s) \xi=T_{u}^{\star}(s) u=T_{\xi}(s) T_{u \rightarrow \xi}(s) u
$$

where $T_{\xi}$ is of degree $k_{\xi}$ and is composed of $T_{u \rightarrow \xi}(s)$ unstable poles. Hence $T_{u}^{\star}(s)$ is a stable transfer of null degree. Using this result, $T_{u}^{\star}$ has a state-space representation $\left(A_{u}^{\star}, B_{u}^{\star}, C_{u}^{\star}, D_{u}^{\star}\right)$ with state $X$ and $D_{u}^{\star} \neq 0$. Moreover, $T_{\xi}(s)$ can be expressed as a monic polynomial of degree $k_{\xi}$ : $T_{\xi}(s)=s^{k_{\xi}}+a_{k_{\xi}-1} s^{k_{\xi}-1}+\ldots+a_{1} s+a_{0}$. It comes

$$
s^{k_{\xi}} \xi=C_{u}^{\star} X+D_{u}^{\star} u-a_{k_{\xi}-1} s^{k_{\xi}-1} \xi-\ldots-a_{1} s \xi-a_{0} \xi
$$

3) OIST: To begin with, we suppose $d_{\Delta}=0$ and $d_{w} \neq 0$. Considering problem 2 , we have to find $\left[u_{\min }(t), u_{\max }(t)\right]$ such that $\forall t, u_{\min }(t) \leq u \leq u_{\max }(t) \Rightarrow \xi_{\min }(t) \leq \xi(t) \leq \xi_{\max }(t)$.

Let $\xi_{n}=T_{\alpha_{n} \rightarrow \xi} \alpha_{n}$. Under Hyp. 1 to 4 we can apply results in Th. 1 (supposing $T_{\alpha_{n} \rightarrow \xi}(s)\left(\Sigma_{1}+\Sigma_{2} s\right.$ ) is proper) thus we obtain saturations on the control signal $u$. These saturations depend on state and state derivative $(x, \dot{x})$ but also on bounds on the wind $\left(\underline{d}_{w}, \bar{d}_{w}\right)$ and its derivative. Two additional problems appear

- in case $(x, \dot{x})$ is not known, this means we can only determine $\widehat{\xi}_{n}=T_{\alpha_{n} \rightarrow \xi} \widehat{\alpha}_{n}$ where $\widehat{\alpha}_{n}=\Sigma_{1} \widehat{x}+\Sigma_{2} \dot{\hat{x}}$. Thus $\widehat{\xi}_{n}$ is also of null relative degree with respect to estimation error $e_{\alpha}^{1}$ on which bounds have been determined in Eq. (34). Indeed we have

$$
\widehat{\xi}_{n}=T_{u \rightarrow \xi}(s) u-T_{\alpha_{n} \rightarrow \xi}(s) e_{\alpha}^{1}
$$

- In case bounds on $d_{w}$ derivatives are not known, we decide to rely on Hyp. 6 in this paper. In future work this hypothesis will be weakened to account for more demanding wind profiles and models.

Remark 1: Under some hypothesis related to our application, we can show that bounding $\xi$ is equivalent to bounding $\alpha$ :

$$
\begin{aligned}
& \alpha_{\min } \leq \underset{\Downarrow^{(1)} \Uparrow^{(2)}}{\alpha} \leq \alpha_{\max } \\
& \xi_{\min } \leq \underset{\xi}{\xi} \leq \xi_{\max }
\end{aligned}
$$


In the presence of uncertainties, $d_{\Delta} \neq 0$ and $\widehat{\xi}_{n}$ is of null relative degree with respect to $d_{\Delta}$ as shown in

$$
\begin{aligned}
\widehat{\xi}_{n} & =T_{u \rightarrow \xi}(s) u-T_{\alpha \rightarrow \xi}(s) e_{\alpha}^{1}+T_{\alpha \rightarrow \xi}(s) \mathcal{B} d_{\Delta} \\
& =T_{u \rightarrow \xi}(s) u-T_{\alpha \rightarrow \xi}(s) e_{\alpha}^{1}-T_{\alpha \rightarrow \xi}(s) e_{\alpha}^{2} \\
& =T_{u \rightarrow \xi}(s) u-T_{\alpha \rightarrow \xi}(s) e_{\alpha}
\end{aligned}
$$

4) Note on the presence of null relative degree inputs in regulated variable expression: Let consider problem 2 and associated notations. In case $\alpha$ also depends on an input $e$ as in $\alpha=C_{\alpha} x+E_{\alpha} e$, expressions proposed in Th. 1 are applied on $\alpha-E_{\alpha} e$ rather than on $\alpha$ which results in modified control saturations since the term

$$
\forall j \in \bar{k}, E_{\alpha} e^{(j)}-\left[\prod_{i=1}^{j}\left(s+K_{i}\right) E_{\alpha} e\right]
$$

is added to the definition of $\alpha_{j, \min }$ and $\alpha_{j, \max }$. This stands as an extension of Th. 1. At this stage control saturations depend on bounds on $e_{\alpha}$ (i.e. on $d_{w}$ and $d_{\Delta}$ bounds), state vector estimate and bounds on derivatives of the estimation error which are discarded in our application using similar hypothesis to Hyp. 6 (see future works).

\section{E. Remarks}

Injecting saturations in a system is never harmless. Consider the case of a dynamic output feedback control law $u=C_{v}(s) y$ (e.g. observer-based controller). Under active saturation, control law is altered into $u=u_{\min }$ or $u=u_{\max }$ which differs from $C_{v}(s) y$. It may result in controller state divergence which is unwanted when switching back from saturation. In that case, consider the use of an anti-windup loop as described for example in [12].

We do not have mentioned the possible presence of positive zeros in transfer $T_{u \rightarrow \xi}$. One may show that a system in closed-loop with active saturating block has its poles equal $T_{u \rightarrow \xi}$ zeros. If positive zeros are present, this means saturated system may diverge away from the saturation. However when absolute value $a$ of a positive zero is very small $a \ll 1$, as is the case in our application, divergence will occur after a long time is spent on saturation, typically of magnitude $\frac{1}{a}$ (in seconds).

\section{APPLICATION}

\section{A. Launch vehicle model}

The model considered is that of a rigid launch vehicle where we also consider sensor dynamics with state $x_{\text {capt }}$. Actuator dynamics is implemented as a second-order filter. The system can be represented by Eq. (5) where $u \in \mathbb{R}$ controls the thruster orientation, $d_{w} \in \mathbb{R}$ is the unknown wind input and $\Theta \in \mathbb{R}^{2}$ are two uncertain parameters related to aerodynamic and thrust coefficients evolution during flight. The state is given by $x=\left(x_{\text {capt }}, \dot{x}_{\text {capt }}, \psi, \dot{\psi}, v_{z}\right)^{\top} \in \mathbb{R}^{5}$ where $\psi$ is the attitude, $\dot{\psi}$ its rate and $v_{z}$ the lateral deviation velocity. We measure $y=\left(\psi, \dot{\psi}, v_{z}\right)^{T^{2}}$. A dynamic controller is synthesized using results in [13].

The considered regulated variable is the angle of attack $\alpha=\psi+\frac{v_{z}-d_{w}}{V}$ where $V$ is the vehicle speed. The angle of

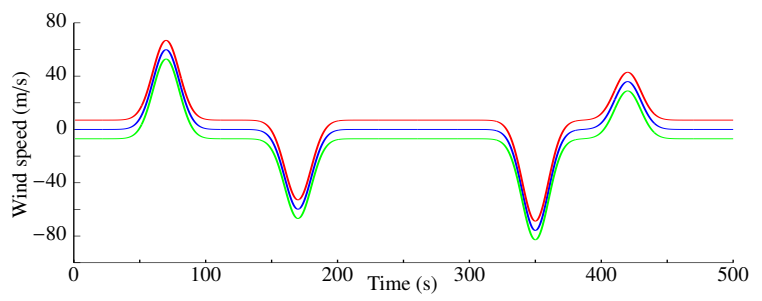

Fig. 3. Wind profile (in blue) and supposed known bounds $\underline{d}_{w}$ and $\bar{d}_{w}$ used for simulation.

attack must remain below a given constant value $|\alpha| \leq \alpha_{\max }$ so as to minimize aerodynamic load during atmospheric flight. Moreover, $\alpha$ depends on the unknown wind input and is not measured. We proceed to decoupling with respect to $d_{w}$ using results in Sect. IV. Transfer $T_{u \rightarrow \alpha_{n}}$ is of relative degree $k=1$ with respect to $u$ but has $n_{\text {unst }}=2$ unstable poles. Thus we proceed to change of variable $T_{\alpha_{n} \rightarrow \xi}=\frac{p_{\xi}}{s+p_{\xi}}$ with $p_{\xi} \gg 1$. Transfer $T_{u \rightarrow \xi}$ is of relative degree $k=2$ with respect to $u$ and $l=1$ with respect to $d_{w}$. Hyp. 2 to 4 are satisfied. Hyp. 1 is not satisfied since an acceptable bound on $\dot{d}_{w}$ cannot be easily determined. In first approach, using Hyp. 6 (satisfied with considered wind profile), we decide to neglect $\dot{d}_{w}$ in the expression of saturations on $u$. We use observers as detailed in Sect. IV to account for incomplete state knowledge.

Note that the matrix $A$ in Eq. (9) is not Metzler. We compute adequate matrix transform $M=R(A-L C) R^{-1}$ so that $M$ is Metzler using reformulation into an equivalent $H_{\infty}$ synthesis problem. This problem is solved using non-smooth $H_{\infty}$ synthesis optimization techniques [14], [15], [16]. A change of coordinates $e_{z}=R e$ is then used as described in Section IV-B.

\section{B. Wind profile, uncertainties and bounds}

We use wind profile presented in Fig. 3 with a $+/-7 \mathrm{~m} / \mathrm{s}$ uncertainty $\left(\underline{d}_{w}, \bar{d}_{w}\right)$ on wind speed. Note this is particularly conservative when considering the launch vehicle control problem since information on the wind speed is much more limited in reality.

Let $d_{\Delta}=\left(d_{\delta_{1}}, d_{\delta_{2}}\right)^{\top}$ the disturbance vector associated to uncertainties $\left(\delta_{1}, \delta_{2}\right)$ on uncertain parameters $\left(\theta_{1}, \theta_{2}\right)$. We denote $\left(\theta_{1, \text { nom }}, \theta_{2, \text { nom }}\right)$ their nominal values. Using Eq. (9) formulation, we find the following relation between $d_{\delta_{i}}$ 's and $\theta_{i}$ 's

$$
d_{\delta_{1}}=-0.1 \delta_{1} \theta_{1, \text { nom }} x_{\text {capt }}, d_{\delta_{2}}=0.4 \delta_{2} \theta_{2, \text { nom }} \alpha
$$

which corresponds to a maximal $\theta_{1, \text { nom }}$ (resp. $\theta_{2 \text {,nom }}$ ) variation of $10 \%$ (resp. $40 \%$ ). We choose to bound uncertainties by

$$
\begin{aligned}
& \left|d_{\delta_{1}}\right| \leq 0.1\left|\theta_{1, \text { nom }} x_{\text {capt }}\right| \\
& \quad\left|d_{\delta_{2}}\right| \leq 0.4 \max \left(\left|\theta_{2, \text { nom }}\left(\widehat{\alpha}+\bar{e}_{\alpha}\right)\right|,\left|\theta_{2, \text { nom }}\left(\widehat{\alpha}+\underline{e}_{\alpha}\right)\right|\right)
\end{aligned}
$$

\section{Method application}

We use results developed in Section IV to

- estimate $\alpha$ as $\widehat{\alpha}$ to be used in $e_{\alpha}$ bounds determination; 

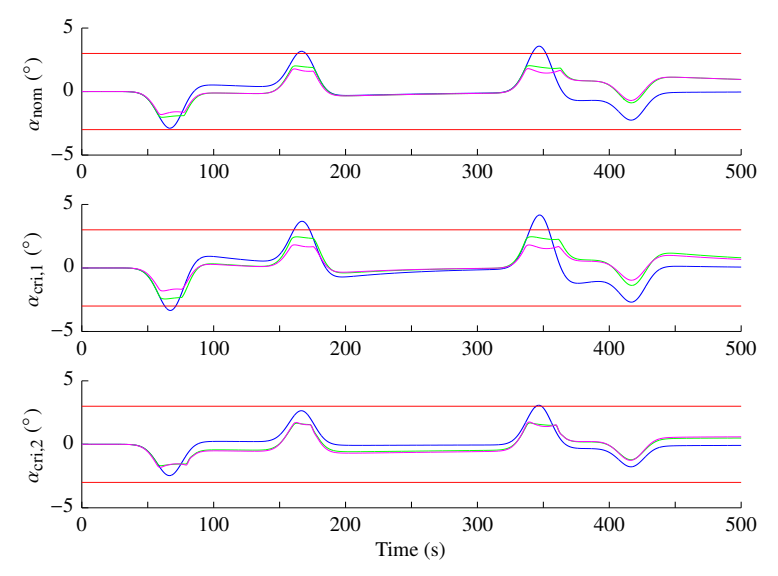

Fig. 4. Unconstrained $\alpha$ (in blue), $\alpha$ with saturations in the loop (in green), $\widehat{\alpha}$ (in magenta) and expected bounds $+/-\alpha_{\max }$ (in red).

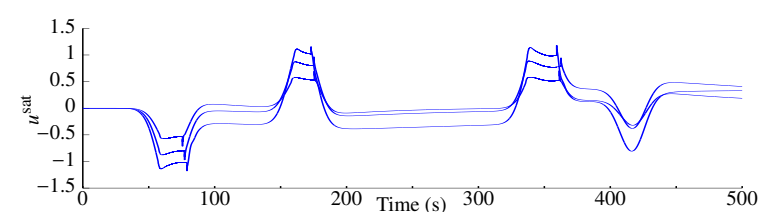

Fig. 5. Obtained saturated control signal in both nominal and critical cases.

- Find bounds $\left(\underline{e}_{\alpha}, \bar{e}_{\alpha}\right)$ on estimation error $e_{\alpha}=\alpha-\widehat{\alpha}$ using interval observer;

- Express time-varying bounds on $\xi$ from which timevarying saturations on control signal $u$ are obtained using Th. 1 applied to the transfer presented in Eq. (38).

Positive parameters $K_{1}$ and $K_{2}$ are chosen static. This requires care in setting bounds on unknown disturbance for the reasons mentioned in III. We use Model Anti-Windup Recovery (MRAW) as described in [12] to avoid divergence of the dynamic controller state upon saturation.

\section{Results}

We perform simulation on nominal model (top) and two representative critical models ${ }^{4}$. Results are shown on Fig. 4 and Fig. 5. Conservativeness appears in the results being more satisfactory than expected since $\alpha$ is often smaller than its maximal expected value. The drawback is that the control signal could be infeasible in practice with physically unacceptable value or rate. This is however not the case with this simplified wind profile.

\section{CONCLUSION}

In this article we have presented a first approach to an effective method to bound an unmeasured disturbed regulated variable $\alpha$ in the case of an uncertain system with unknown disturbance input. We have used a combination of interval observer and OIST method to circumscribe estimation error and robustly bound acceptable control signals. We have used an existing controller synthesized using linear control

\footnotetext{
${ }^{4}$ That is $\left(\delta_{1}=1, \delta_{2}=-1\right)$ and $\left(\delta_{1}=-1, \delta_{2}=1\right)$.
}

technique according to given requirements. Our approach allows to enforce output constraint requirements which may compete with synthesis requirements. Recently proposed OIST method [3] proposes to transform expected bounds on $\alpha$ into saturations on the control $u$. When no saturation is active, the behaviour is that of the chosen controller. Care must be taken in case of a dynamic controller since its state may diverge under active saturations. Coupled to interval observer, we show this method can be applied to uncertain systems with unknown disturbance. This approach has been successfully applied to a rigid launch vehicle with five states, two uncertain parameters and one unknown disturbance (wind input).

To be able to use results detailed in Section III we have made restrictive hypotheses on our model. Static $\left(K_{1}, K_{2}\right)$ have been chosen which lead to conservative results. Wind profile and known bounds are also not realistic. These limits will be considered in future works. Note that this article may be considered as a proof of feasibility while not considering issues of stability or zero-dynamics which are under extensive study at the moment.

$$
\begin{aligned}
& \text { ApPendix A }
\end{aligned}
$$

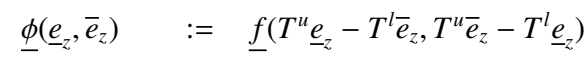

$$
\begin{aligned}
& \bar{\phi}\left(\underline{e}_{z}, \bar{e}_{z}\right) \quad:=\bar{f}\left(T^{u} \underline{e}_{z}-T^{l} \bar{e}_{z}, T^{u} \bar{e}_{z}-T^{l} \underline{e}_{z}\right) \\
& \underline{f}^{z}\left(\underline{e}_{z}, \bar{e}_{z}\right) \quad:=R^{u} \underline{\phi}\left(\underline{e}_{z}, \bar{e}_{z}\right)-R^{l} \bar{\phi}\left(\underline{e}_{z}, \bar{e}_{z}\right) \\
& \bar{f}^{z}\left(\underline{e}_{z}, \bar{e}_{z}\right) \quad:=R^{u} \bar{\phi}\left(\underline{e}_{z}, \bar{e}_{z}\right)-R^{l} \underline{\phi}\left(\underline{e}_{z}, \bar{e}_{z}\right) \\
& \underline{B_{e}^{z}}(\widehat{x}, u) \quad:=R^{u} \underline{B_{e}}(\widehat{x}, u)-R^{l} \overline{B_{e}}(\widehat{x}, u) \\
& \overline{B_{e}^{z}}(\widehat{x}, u) \quad:=R^{u} \overline{B_{e}}(\widehat{x}, u)-R^{l} \underline{B_{e}}(\widehat{x}, u) \\
& \begin{aligned}
\underline{B}_{d}^{z}\left(\underline{d}_{w}, \bar{d}_{w}\right):= & R^{u} \min \left(\underline{B_{d}}\left(\bar{d}_{w}\right) \bar{d}_{w}, \underline{B}_{d}\left(\underline{d}_{w}\right) \underline{d}_{w}\right) \\
& -R^{l} \max \left(\bar{B}_{d}\left(\bar{d}_{w}\right) \bar{d}_{w}, \bar{B}_{d}(\underline{d}) d\right.
\end{aligned} \\
& \overline{B_{d}^{z}}\left(\underline{d}_{w}, \bar{d}_{w}\right):=R^{u} \max \left(\overline{B_{d}}\left(\bar{d}_{w}\right) \bar{d}_{w}, \overline{B_{d}}\left(\underline{d}_{w}\right) \underline{d}_{w}\right) \\
& -R^{l} \min \left(\underline{B_{d}}\left(\bar{d}_{w}\right) \bar{d}_{w}, \underline{d}_{d}\left(\underline{d}_{w}\right) \underline{d}_{w}\right)
\end{aligned}
$$

\section{REFERENCES}

[1] O. J. Rojas and G. C. Goodwin, "A simple anti-windup strategy for state constrained linear control," in Proc. of the 15th IFAC World Congress, Barcelona, Spain, 2002.

[2] M. C. Turner and I. Postlethwaite, "Output violation compensation for systems with output constraints," IEEE TAC, vol. 47, no. 9, pp. 1540-1546, 2002.

[3] L. Burlion, "A new saturation function to convert an output constraint into an input constraint," in Proc. of the Mediterranean Conference on Control and Automation, Barcelona, Spain, July 2012, pp. 1218-1222.

[4] F. Mazenc and O. Bernard, "Interval observers for linear time-invariant systems with disturbances," Automatica, vol. 47, no. 1, pp. 140-147, Jan. 2011.

[5] D. Efimov, T. Raïssi, and A. Zolghadri, "Control of nonlinear and LPV systems: Interval observer-based framework," IEEE Transactions on Automatic Control, vol. 58, no. 3, pp. 773-778, Mar. 2013.

[6] L. Burlion and H. de Plinval, "Keeping a ground point in the camera field of view of a landing UAV," in Proc. of the IEEE International Conference on Robotics and Automation, 2013, pp. 5763-5768.

[7] L. Burlion, C. Poussot-Vassal, P. Vuillemin, M. Leitner, and T. Kier, "Longitudinal manœuvre load control of a flexible large-scale aircraft," in Proc. of the 19th IFAC World Congress, 2014, pp. 3413-3418.

[8] J. C. Doyle, "Structured uncertainty in control system design," in Proc. of 24th IEEE Conference on Decision and Control, Ft. Lauderdale, FL, Dec. 1985, pp. 260-265. 
[9] L. K. Vasiljevic and H. K. Khalil, "Differentiation with high-gain observers in presence of measurement noise," in Proc. of the 45th IEEE Conference on Decision and Control, San Diego, CA, USA, Dec. 2006, pp. 4717-4721.

[10] J. Reger and J. Jouffroy, "On algebraic time-derivative estimation and deadbeat state reconstruction," in Proc. of the Joint 48th IEEE Conference on Decision and Control and 28th Chinese Control Conference, Shangai, P.R., China, Dec. 2009, pp. 1740-1745.

[11] T. N. Dinh, F. Mazenc, and S.-I. Niculescu, "Interval observer composed of observers for nonlinear systems," in Proc. of the European Control Conference, Strasbourg, France, June 2014, pp. 660-665.

[12] F. Forni, S. Galeani, and L. Zaccarian, "Model recovery anti-windup for continuous-time rate and magnitude saturated linear plants," Automatica, vol. 48, no. 8, pp. 1502-1513, Aug. 2012.

[13] O. Voinot, D. Alazard, and A. Piquereau, "A robust multi-objective synthesis applied to launcher attitude control," in Proc. of the 15th IFAC Symposium on Automatic Control in Aerospace, Sept. 2001.

[14] P. Apkarian, "Tuning controllers against multiple design requirements," in Proc. of the American Control Conference, Washington, USA, June 2013, pp. 3888-3893.

[15] P. Apkarian and D. Noll, "Nonsmooth $H_{\infty}$ synthesis," IEEE Transactions on Automatic Control, vol. 51, no. 1, pp. 71-86, Jan. 2006.

[16] J. V. Burke, D. Henrion, A. S. Lewis, and M. L. Overton, "HIFOO - a MATLAB package for fixed-order controller design and $H_{\infty}$ optimization," in Proc. of the 5th IFAC Symposium on Robust Control Design, Toulouse, France, Aug. 2006. 